\title{
Factores de riesgo de bronquiolitis en niños menores de un año, hospital Luis Fernando Martínez, Cañar entre enero y julio del 2018
}

\section{Risk factors for bronchiolitis in children under one year of age, Luis Fernando Martínez Hospital, Cañar between January and July 2018}

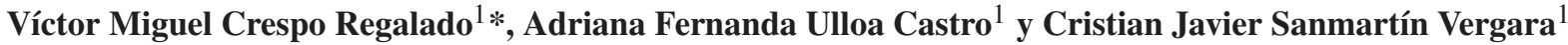 \\ ${ }^{1}$ Universidad Católica de Cuenca \\ *vmcrespor@ucacue.edu.ec
}

DOI: https://doi.org/10.26871/killkana_salud.v3i3.523

\begin{abstract}
Resumen
Contexto: La bronquiolitis, es una de las patologías más habituales en la infancia, resultando ser la principal causa de ingreso a nivel hospitalario en los niños menores de 2 años con infección del tracto respiratorio inferior. Se caracteriza por presentar síntomas respiratorios que van progresando paulatinamente, para luego debutar con síntomas de las vías respiratorias inferiores como sibilancias, tiraje intercostal, disnea, etc, por la infección primaria o la re-infección con el virus Sincitial Respiratorio que es uno de los patógenos más frecuentes. Objetivo: El objetivo principal del estudio fue evaluar los factores de riesgos de bronquiolitis en el Hospital Luis Fernando Martínez de la ciudad de Cañar, en el periodo comprendido entre enero y julio del 2018. Procedimientos: El tipo de estudio es descriptivo trasversal, se aplica la escala de Wood Downes para el diagnóstico, y se realiza la correspondiente tabulación de datos, presentándolos en cuadro de respuesta a los objetivos. Resultados: La bronquiolitis afecta más al sexo masculino que al femenino, la edad predominante entre los 9 y 10 meses de edad, no existe significancia de factores de riesgo como humo de cigarrillo o condiciones socioeconómicos para la enfermedad, sin embargo, la lactancia materna eleva en tres veces el riesgo de contraer la enfermedad con riesgo de muerte por bronquiolitis, siendo importante su exclusividad hasta alcanzar el sexto mes de vida.
\end{abstract}

Palabras clave: bronquiolitis, lactancia materna, factores socioeconómicos, niños lactantes.

\begin{abstract}
Context: Bronchiolitis is one of the most common pathologies in childhood, proving to be the main cause of hospital admission in children under 2 years of age with lower respiratory tract infection. It is characterized by presenting respiratory symptoms that are progressing gradually, and then debuting with symptoms of the lower respiratory tract such as wheezing, intercostal circulation, dyspnea, etc., due to primary infection or re-infection with the Respiratory Syncytial virus, which is one of the most frequent pathogens. Objective: The main objective of the study was to evaluate the risk factors of bronchiolitis at the Luis Fernando Martinez Hospital in the city of Cañar, in the period between January and July 2018. Procedures: The type The study is cross-sectional, the Wood Downes scale is applied for diagnosis, and the corresponding data tabulation is performed, presenting them in a response box to the objectives. Results: Bronchiolitis affects more male than female sex, the predominant age between 9 and 10 months of age, there is no significance of risk factors such as cigarette smoke or socioeconomic conditions for the disease, however, Breastfeeding increases the risk of contracting the disease at risk of death from bronchiolitis by three times, its exclusivity being important until reaching the sixth month of life.
\end{abstract}

Keywords: bronchiolitis, breastfeeding, economic factors, nursing children.

\section{Introducción}

En un estudio realizado por G. Orejón de Luna, M. Fernández Rodríguez se menciona que una de las patologías respiratorias más frecuentes es la bronquiolitis aguda en la infancia, con un porcentaje anual de incidencia aproximado del $10 \%$ en pacientes niños que son menores a los 2 años. Esta situación produce una importante demanda de pacientes que acuden a la atención de salud de primer nivel con 
una frecuencia de consultas que varía entre $4 \%$ y el $20 \%$; además en las áreas hospitalarias de urgencias presentan una frecuencia de consultas que varían 0.8 al $2.5 \%$. La infección del tracto respiratorio inferior es la causa más frecuente de ingreso hospitalario de tipo agudo en niños menores de 2 años, con una frecuencia de ingreso del $1 \%$ y el $5 \% .^{1}$

En un estudio realizado por la Dra. Andrea Parra, Dra. Carolina Jiménez, Dra. Sara Hernández, Dr. Jorge Edwin García, Dra. Ángela María Cardona dice que los principales factores de riesgo que extienden la posibilidad de poseer bronquiolitis destaca la decisión de madres a dejar sus niños a guarderías, poseer hermanos mayores que comparten el mismo cuarto o dormitorio, la lactancia materna menor a los dos meses, familiares o padres con hábitos de tabaco, sobre todo si se tiene la exposición a tabaco durante el periodo de gestación, tener bajo peso al nacer, ser de sexo masculino, el nivel socioeconómico bajo, y el hacinamiento.

Los principales factores que pueden progresar dicha patología a severa es la EPOC, cardiopatías de carácter congénito, ser prematuro, edades inferiores a los tres-seis meses, displasia broncopulmonar, la inmunodeficiencia, enfermedad neurológicas, y los defectos anatómicos de las vías respiratorias ${ }^{2}$

C. Ochoa, J. González en su estudio realizado mencionan que las más importantes identificaciones clínicas verificadas han sido organizadas por orden de frecuencia, es decir, la tos con un porcentaje del $99.8 \%$, seguido de la rinitis $80.7 \%$, rechazo de las tomas en un $37 \%, 19,6 \%$ en la fiebre de 38 grados centígrados, vómitos en un $21.4 \%$. Es extraño la presentación de deshidratación en un $0.7 \%$ y apneas en un $1.8 \%$, quienes poseían una calificación en la escala de gravedad media de 1.45 , presentando más de 4 puntos solo el $4,4 \% .^{3}$

Dr. Benito Javier, jefe del Servicio de Urgencias del Hospital Universitario de Cruces en el área de Pediatría dice que entre el 15 o $20 \%$ de los niños con bronquiolitis son hospitalizados, especialmente los menores de seis meses de edad. Solo en Bizkaia, entre noviembre de 2014 y enero de 2015 fueron atendidos en las urgencias hospitalarias alrededor de 1.500 episodios de bronquiolitis, siendo hospitalizados más de 200 niños con esta enfermedad.

La fase aguda de la enfermedad dura entre 7 y 12 días e independientemente de la gravedad del episodio de bronquiolitis, entre un 35 y $50 \%$ de los pacientes presentará en el futuro episodios recurrentes de sibilancias y dificultad respiratoria. La morbi-mortalidad de la enfermedad ha disminuido los últimos años, a raíz de los avances en los cuidados hospitalarios de estos pacientes y se concentra en específicos en niños con factores de riesgo.

La prematuridad, las enfermedades respiratorias crónicas, el síndrome de Down, enfermedades neuromusculares, enfermedades cardíacas congénitas, inmunodeficiencia y el síndrome velo-cardio-facial son fuertes predictores de mortalidad intrahospitalaria causada por bronquiolitis. El impacto social, económico y sanitario es por lo tanto muy relevante y se prolonga más allá de la fase aguda ${ }^{4}$

Richard Baquero Rodríguez y Arturo Granadillo Fuentes dice que el virus sincitial respiratorio en la bronquiolitis presenta un período de desarrollo que va de los dos a los ocho días muestra replicaciones en la nasofaringe a nivel de su epitelio, diseminándose y afectando el epitelio en las vías respiratorias inferiores en el tiempo que va de uno a los tres días a nivel de las vías aéreas de menos calibre causa necrosis en el epitelio, lo que acompañado a la respuesta, se asocia el edema en la submucosa y producción de moco, siendo uno de los principales responsables de producir la hiper-insuflación, las atelectasias y las sibilancias manifestadas en los niños.

A los pocos días comienza la mejoría a nivel histológica pero la recuperación completa puede tardar varias semanas. En la neumonía causada por el Virus Sincitial Respiratorio (VSR) hay células mononucleares con infiltrados intersticiales acompañados de hipertrofia de la pared muscular de los bronquiolos. ${ }^{5}$

En la Escuela de Medicina de la Universidad de Washington, en Missouri, varios expertos realizan un estudio donde revela que si la madre acostumbra a fumar o el consumo de cigarrillos después del nacimiento indica una gravedad de la enfermedad, es decir, la bronquiolitis en los niños menores de 2 años acompañado con las que consumieron tabaco en una forma pasiva, los niños de edades menores presentaron una saturación de oxígeno menor, resultado que indica una mayor dificultad al momento de respirar y, por lo consecuente, una mayor complicación de la patología.

La existencia de varios casos de alergias en las familias, en especial de la madre y al ser de raza negra representaron ser dos factores importantes que pueden proporcionar que la bronquiolitis sea menos grave. En cambio, la exposición a humo de tabaco y la edad temprana en niños están asociados con el aumento de la gravedad de la bronquiolitis, mientras que las madres que no son alérgicas podrían tener un carácter protector sobre dicha patología.

Según estudio realizado Perdikidis Olivieri L, Suwezda A. menciona que en el estudio realizado a 619 niños se ven los factores de riesgo teniendo con un mayor porcentaje a la exposición a cigarrillos en un $65 \%$ de sexo masculino. Se registró un reingreso de niños que tenían seguimiento durante un año con un porcentaje de un $17 \%$ con proporciones del EHTA del $35 \%$ - $56 \%$ y el $79 \%$ según la evaluación realizada.

La cotinina que está en el suero y la saliva están ampliamente asociadas. Entre la cotinina que se detectó en suero o saliva, y el porcentaje de los reingresos hospitalarios se presenta un cambio en cuanto a la exposición de tabaco; revelada por el nivel socioeconómico. No hay detecciones asociadas a la exposición de humo de tabaco mencionada por cuidadores y a nivel de hospitalarios en sus ingresos ${ }^{6}$

L. Pérez Cid, B. San José Valiente, V. Quintero Calcaño recalcan que la leche materna tiene mucha importancia, en 
especial para los niños que se encuentran en los primeros meses de vida y los que nacieron prematuramente pues contiene componentes esenciales que trasformados una vez en el organismo evitan enfermedades respiratorias y baja el número en cuatro veces el riesgo de muerte por presentar bronquiolitis.

La lactancia materna ofrece una gran cantidad de factores que ayudan a la maduración, el crecimiento de varios mecanismos que favorecen a la defensa que posee y desarrolla el organismo, presentando un bajo desarrollo de las estructuras principales de funciones afines con la función de respirar.

La lactancia materna en lactantes y neonatos, presenta un déficit en el desarrollo de presentar bronquiolitis y neumonía, y baja el grado de gravedad de los cuadros en niños que los adquieren habiendo tenido lactancia materna exclusiva durante los seis meses de vida. Una buena estrategia para reforzar el sistema inmunitario del bebé y hacerle más fuerte ante ella, es prolongar más allá de los cuatro meses, preferiblemente durante los primeros seis meses. ${ }^{7}$

La presente investigación se enfocará en poder aplicar la escala de Wood Downes, misma que es útil en el diagnóstico de la bronquiolitis en pacientes menores de un año. Dicha escala es de alto grado de confiabilidad, y fácil aplicación según se evidencia en muchos trabajos elaborados.

El proyecto investigativo resulta de mucha importancia ya que en los últimos años la patología conocida como la bronquiolitis, en las poblaciones menores de 1 año presenta un intervalo de $1.3-5 \%$ de los ingresos hospitalarios por bronquiolitis aguda. ${ }^{3}$

Teniendo como una de las principales causas de la bronquiolitis aguda al Virus Sincitial Respiratorio considerados en estos últimos años como epidemias, no solo a nivel de Latinoamérica sino a nivel mundial, este proyecto ayudará a conocer la realidad del entorno; es común que los niños presenten este tipo de enfermedades principalmente en épocas de invierno, por lo que se descubrirá cuáles son los factores de riesgo que generan esta patología para a su vez diagnosticar con mayor precisión y prevenir en la población futuras complicaciones.

\subsection{Objetivo General}

Evaluar los factores de riesgos de bronquiolitis en el Hospital Luis Fernando Martínez durante el periodo de enero a julio del año 2018.

\subsection{Objetivos Especificos}

Identificar el género más frecuente en el que se presenta la bronquiolitis.

Estudiar cuál es la edad de mayor riesgo para presentar la enfermedad.

Identificar el tratamiento más utilizado para la bronquiolitis.

Aplicar la escala de Wood Downes y realizar la correspondiente tabulación de datos.
Determinar el porcentaje de pacientes con bronquiolitis atendidos en el área de Pediatría.

\section{Materiales y Métodos}

El tipo de estudio fue descriptivo y trasversal. Se realizó en el servicio de Pediatría del Hospital Luis Fernando Martínez de la ciudad de Cañar durante el periodo enero a julio del año 2018. El universo constituyeron todos los pacientes menores de 1 año con diagnóstico de bronquiolitis que fueron ingresados en el departamento de Pediatría, en el periodo, tanto de sexo masculino como femenino. La muestra estuvo compuesta de cien pacientes de entre 0 a 12 meses de edad con diagnóstico de bronquiolitis y complicaciones en el hospital en el periodo referido.

Una vez obtenida la información se transcribió a una base de datos digital para su respectiva tabulación y organización. Para el procesamiento de los datos se utilizó la Escala de Wood Downes que es una escala de evaluación, utilizada fundamentalmente en la valoración de la gravedad de las bronquiolitis, aunque también se ha utilizado para valoración del asma agudo, sobre todo en el ámbito infantil y permite un tratamiento oportuno de los pacientes, realizándose además la revisión de la historia clínica del paciente. Los resultados están presentados mediante gráficos y tablas, según se cree pertinente de acuerdo al uso de la estadística descriptiva.

Fueron criterios de inclusión: menores de 1 año de edad, pacientes con diagnóstico de bronquiolitis al egreso, sexo indistinto, padres que aceptaron que sus hijos participen en la investigación. Fueron criterios de exclusión: pacientes mayores de 2 años y niños cuyos padres no aceptaron que se los incluya en la investigación.

Para la investigación se incluyeron aspectos bioéticos, fundamentados en los principios de autonomía, beneficencia y justicia. Se elaboró un formato de consentimiento informado para el examen físico respiratorio, y los exámenes complementarios requeridos.

\section{Resultados}

Fig. 1. Pacientes que presentan bronquiolitis según su sexo de enero-julio del 2018

Según el estudio, del total de pacientes con bronquiolitis, $67 \%$ son del sexo masculino y $33 \%$ del sexo femenino, 
lo cual evidencia que la enfermedad es predominante según sexo, concordante con los resultados obtenidos en el estudio, Guía Práctica Clínica: Bronquiolitis, de Richard Baquero Rodríguez y Arturo Granadillo Fuentes ${ }^{5}$ en el que se encontró predominancia de la enfermedad en niños de sexo masculino.

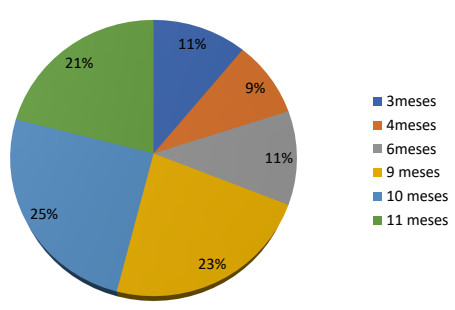

Fig. 2. Pacientes con diagnóstico de bronquiolitis por el grupo de edad

Del total de pacientes estudiados (100) con diagnóstico de bronquiolitis según la edad, el $25 \%$ corresponde a pacientes de 10 meses de edad, el $23 \%$ corresponde a pacientes de 9 meses de edad, el $21 \%$ corresponde a pacientes de 11 meses, el $11 \%$ corresponde a pacientes con 6 y 3 meses de edad y el $9 \%$ a pacientes de 4 meses de edad.

Si se observa el gráfico 2, se puede deducir que el $69 \%$ aproximadamente de los pacientes, presentan la enfermedad entre los 9 y los 11 meses, siendo este grupo etario el más susceptible de padecer la enfermedad, en discrepancia con el estudio de Richard Baquero Rodríguez y Arturo Granadillo Fuentes, ${ }^{5}$ en el cual la edad de predominancia de la bronquiolitis estuvo entre los 3 y 6 meses, y coincidente con el estudio de bronquiolitis en el Servicio de Pediatría del Hospital de la «Santa Creu i Sant Pau» de Barcelona, España, en el cual el $42 \%$ de los pacientes se encuentra entre los 4 y 8 meses de edad.

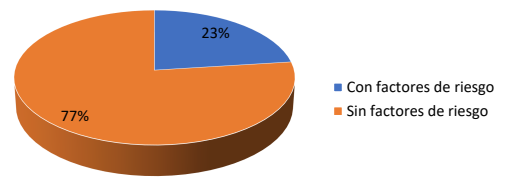

Fig. 3. Pacientes con diagnóstico de bronquiolitis según el factor de riesgo

El $77 \%$ de pacientes con diagnóstico de bronquiolitis no presento relación alguna con los factores de riesgo estudiados como prematuridad, las enfermedades respiratorias crónicas, el síndrome de Down, enfermedades neuromusculares, enfermedades cardíacas congénitas, inmunodeficiencia y el síndrome velo-cardio-facial, solo el $23 \%$ de pacientes se asoció a algún factor de riesgo.

De alguna manera el estudio concuerda con el artículo del Dr. Raúl R. Esquivel S. Factores de riesgo para el desarrollo de bronquiolitis severa en niños menores de 2 años admitidos al Hospital del Niño, Panamá de diciembre de 2013 a abril de $2014 .{ }^{8}$ En este trabajo se logró documentar asociación entre la prematuridad menor de 32 semanas, la presencia de co-morbilidad, el bajo peso, convivencia con niños menores de cinco años en el hogar, aunque no se encontró asociación entre la edad menor o igual a dos meses, la etnia indígena, tabaquismo pasivo, antecedente de asma en la madre, infección por VRS ni co-infección viral.

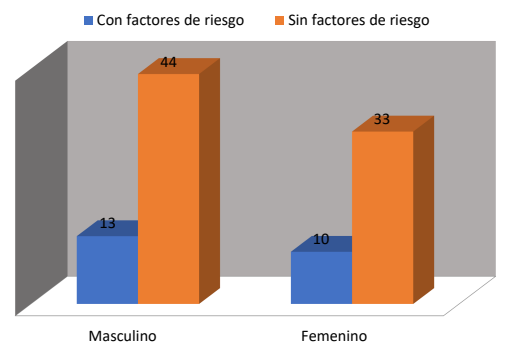

Fig. 4. Pacientes con diagnóstico de bronquiolitis según el factor de riesgo y género

De todos los pacientes con diagnóstico de bronquiolitis, el $77 \%$ no tiene factores de riesgo evidente, de los cuales 44 son varones y 33 mujeres. Mientras que de los 23 pacientes que tuvieron bronquiolitis asociado con algún factor de riesgo, el $56 \%$ son varones y el $44 \%$ mujeres, lo que evidencia que los factores de riesgo no se asocian de modo significatico al sexo de los pacientes con la enfermedad.

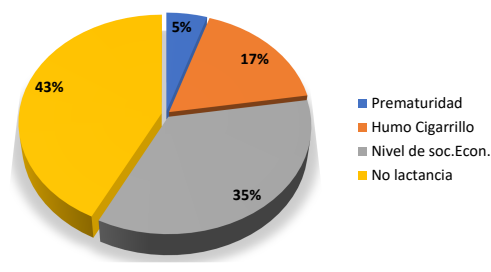

Fig. 5. Pacientes con diagnóstico de bronquiolitis según los factores de riesgo

De los pacientes con diagnóstico de bronquiolitis, que fueron asociados a factores de riesgo (23), el $8,7 \%$ fue vinculado a diferentes grados de prematuridad, el 30,4\% estuvo asociado a exposición al humo del cigarrillo, el $60,9 \%$ se relacionó con nivel socioeconómico bajo y el $74 \%$ estuvo motivado por ausencia de lactancia materna. 


\section{Discusión}

El presente estudio concuerda en que el sexo predominante para el desarrollo de bronquiolitis es el masculino en una proporción de 2 a 1, encontrado por Richard Baquero Rodríguez y Arturo Granadillo Fuentes, publicado en Guía Práctica Clínica: Bronquiolitis. ${ }^{5}$

El $69 \%$ aproximadamente de los niños presentan la enfermedad entre los 9 y los 11 meses, siendo este grupo etario para el criterio de los autores el más susceptible de padecer la enfermedad, en coincidencia con el estudio de Bronquiolitis en el Servicio de Pediatría del Hospital de la «Santa Creu i Sant Pau» de Barcelona, España, en el cual el $42 \%$ de los pacientes se encuentra entre los 4 y 8 meses de edad. ${ }^{9}$

El $77 \%$ de pacientes que fueron diagnosticados de bronquiolitis, no tuvieron asociación significativa con factores de riesgo. Del $23 \%$ que sí lo tuvieron, la mayor parte de ellos fueron asociados a la no lactancia materna, nivel socio económico bajo y exposición al humo de cigarrillo, y concuerda plenamente con el estudio del Dr. Raúl R. Esquivel S. Factores de riesgo para el desarrollo de bronquiolitis severa en niños menores de 2 años admitidos al Hospital del Niño, Panamá. ${ }^{8}$

\section{Conclusiones}

A pesar de que la casuística presentada no es muy grande, la muestra es significativa, lo que permite realizar algunas conclusiones que se pueden inferir a poblaciones de similares características.

La enfermedad es altamente predominante en niños del sexo masculino, coincidente con otros estudios latinoamericanos.

Las dos terceras partes de pacientes con bronquiolitis se encuentran entre la edad de los 9 y 11 meses, lo cual es importante si se consideran las complicaciones como bronconeumonía o neumonías virales o bacterianas que son mejor manejadas en este grupo etario.

Se demostró en el estudio que los supuestos factores de riesgo no son determinantes en producir la enfermedad, sin embargo, en aquellos casos que fueron ligados a dichos factores, la ausencia de lactancia materna, la baja condición socio económica familiar y la contaminación por humo de cigarrillo estuvieron entre los principales factores.

Es necesario que futuros estudios determinen las posibilidades genéticas de contraer la enfermedad, toda vez que al ser una patología frecuente, requiere mayor dedicación para evitar la alta tasa de mortalidad infantil que aún prevalece en el país.

\section{Fuente de Financiamiento}

Este estudio es autofinanciado.

\section{Conflicto de Intereses}

No existen conflictos personales, profesionales, financieroso de otro tipo.

\section{Consentimiento Informado}

Los autores cuentan con el consentimiento informado de los pacientes para la investigación, la publicación del caso y sus imágenes.

\section{Referencias Bibliográficas}

1. Orejon de Luna M, Fernández Rodríguez M. Bronquiolitis Aguda. Pediatric Atencion Primaria. 2012 Jun;14:167176.

2. Parra A, Jiménez C, Hernández S. Bronquiolitis. Neumología Pediátrica. 2013;8:95-101.

3. Ochoa Sangrador C, González de Dios J. Manejo de bronquiolitis aguda en atención primaria. AN Pediatric. 2013;79:167-179.

4. Benito J. Bronquiolitis aguda por Virus SincitiaL; 2015.

5. Baquero Rodríguez R, Granadillo Fuentes A. Bronquiolitis. Guía de Práctica Clínica. 2009;

6. Perdikidis Olivieri L. La exposición al tabaco se relaciona con los reingresos por asma en niños. Evidencia Pediatrica. 2014;

7. Martínez-Baylach J, Castán AR, Rieró JC. Estudio clínico y epidemiológico de la bronquiolitis aguda en pacientes menores de un año de edad. Acta Pediatrica Espanola. 2004;62(7):275.

8. Esquivel RR. Factores de riesgo para el desarrollo de bronquiolitis severa en niños menores de 2 años admitidos al Hospital del Niño, Panamá diciembre de 2013 a abril de 2014. Pediátr Panamá. 2016;p. 26-30.

9. Garcia Garcia M, Korta Murua J, Callejon Callejon A. Bronquiolitis Aguda Viral. Protocolo Diagnostic Ter Pediatric. 2017;p. 85-102.

Recibido: 10 de abril de 2019

Aceptado: 2 de septiembre de 2019 
